12,13

\title{
О диполь-дипольном взаимодействии атомов в слоях, адсорбированных на трехмерных и двумерных полупроводниках
}

\author{
() С.Ю. Давыдов ${ }^{1}$, А.В. Зубов ${ }^{2}$ \\ ${ }^{1}$ Физико-технический институт им. А.Ф. Иофрфе РАН, \\ Санкт-Петербург, Россия \\ ${ }^{2}$ Санкт-Петербургский национальный исследовательский университет \\ информационных технологий, механики и оптики (ИТМО), \\ Санкт-Петербург, Россия \\ E-mail: Sergei_Davydov@mail.ru \\ Поступила в Редакцию 17 марта 2020 г. \\ В окончательной редакции 17 марта 2020 г. \\ Принята к публикации 23 апреля 2020 г.
}

\begin{abstract}
Предложены простые модельные схемы учета диполь-дипольного отталкивания адатомов в субмонослоях на полупроводниковых трех- и двумерных подложках. Приведены общие выражения для определения вкладов зонных и локальных состояний в переход заряда между адсорбированном слоем и подложкой в функции от степени покрытия подложки адатомами. Аналитические оценки сделаны для ряда частных характерных случаев.
\end{abstract}

Ключевые слова: адсорбция, переход заряда, диполь-дипольное отталкивание, зонные и локальные состояния.

DOI: $10.21883 /$ FTT.2020.08.49618.061

\section{1. Введение}

При адсорбции атомов на твердотельных подложках нужно учитывать их взаимодействие. Самыми важными каналами такового являются диполь-дипольное взаимодействие, косвенный обмен через электронные состояния подложки и прямой обмен при высоких концентрациях адатомов [1-3]. Диполь-дипольное взаимодействие возникает, когда адатомы имеют заряд, возникший вследствие перехода электронов (дырок) с адатома в подложку. Именно этот вид взаимодействия будет рассмотрен в настоящей статье.

Для атомов, адсорбированных на металле, дипольдипольное отталкивание адекватно описывается моделью Муската-Ньюнса [4] (см. также [2,3]). В этой модели рассматривается решетка параллельных диполей, образованных заряженными адатомами и их изображениями в подложке. При этом речь идет об адсорбции одинаковых атомов, занимающих одинаковые адсорбционные позиции. Отметим, что модель Муската-Ньюнса основана на использовании гамильтониана Андерсона $[2,3,5-7]$.

Согласно модели Муската-Ньюнса [2-4], число заполнения $n_{a}$ одноэлектронного (однодырочного) адатома может быть определено из самосогласованного уравнения

$$
n_{a}(\Theta)=\frac{1}{\pi} \operatorname{arcctg} \frac{\varepsilon_{a}-\Theta^{3 / 2} \xi Z_{a}(\Theta)-E_{\mathrm{F}}}{\Gamma} .
$$

Здесь $\Theta=N_{a} / N_{M L}-$ степень покрытия, где $N_{a}$ и $N_{M L}$ - двумерные концентрации адатомов в субмо- нослое и монослое, $0 \leq \Theta \leq 1, \varepsilon_{a}-$ энергия уровня адатома, $E_{\mathrm{F}}-$ уровень Ферми, $Z_{a}(\Theta)$ - заряд адатома, равный $1-n_{a}(\Theta)$, если уровень адатома изначально (до адсорбции) заполнен (адатомы-доноры), и $-n_{a}(\Theta)$, если уровень адатома изначально пуст (адатомы-акцепторы), $\Gamma=$ const - полуширина квазиуровня адатома, $\xi=2 e^{2} l^{2} N_{M L}^{3 / 2} A-$ константа дипольдипольного взаимодействия, $e-$ заряд электрона, $l-$ длина адсорбционной связи, $A \sim 10-$ множитель, слабо зависящий от геометрии адслоя [8].

В случае адсорбции на полупроводнике дело обстоит не столь просто, так как в формировании заряда адатома участвуют не только зонные состояния $n_{b}$ (как это имеет место в случае металлической подложки), но и локальные состояния адсорбционной системы $n_{l}$, лежащие в запрещенной зоне подложки $[2,3]$. Та же ситуация имеет место и при адсорбции на графеноподобных соединениях [9]. Именно такие подложки мы и рассмотрим в настоящей работе.

\section{2. Общие соотношения}

В рамках модельного подхода к проблеме адсорбции $[2,3]$, плотность состояний на одиночном адатоме $\rho_{a}(\omega)$ может быть представлена в виде

$$
\rho_{a}(\omega)=\frac{1}{\pi} \frac{\Gamma(\omega)}{\left[\omega-\varepsilon_{a}-\Lambda(\omega)\right]^{2}+\Gamma^{2}(\omega)} .
$$

Здесь $\omega-$ энергетическая переменная, $\Gamma(\omega)=$ $=\pi V^{2} \rho_{\text {sub }}(\omega)-$ функция уширения квазиуровня, где 
$\rho_{s u b}(\omega)$ - плотность состояний подложки, $V-$ матричный элемент взаимодействия адатом-подложка, и $\Lambda(\omega)$ - функция сдвига квазиуровня (гильбертова трансформанта функции $\Gamma(\omega))$. Выражение (2) легко переписать на случай конечных покрытий, заменив $\rho_{a}(\omega)$ на $\rho_{a}(\omega, \Theta)$ и $\varepsilon_{a}$ на $\varepsilon_{a}(\Theta)=\varepsilon_{a}-\varepsilon_{d i p}(\Theta)$, $\varepsilon_{d i p}(\Theta)=\Theta^{3 / 2} \xi Z_{a}(\Theta)$. При нулевой температуре вклады в суммарное число заполнения $n_{a}(\Theta)$ валентной зоны $n_{v}(\Theta)$ и локального состояния $n_{l}(\Theta)$ равны соответственно

$$
\begin{aligned}
& n_{v}(\Theta)=\int_{-\infty}^{E_{V}} \rho_{a}(\omega, \Theta) d \omega, \\
& n_{l}(\omega)=\left|1-\frac{d \Lambda(\omega)}{d \omega}\right|_{\omega_{l}(\Theta)}^{-1},
\end{aligned}
$$

где $E_{V}$ - энергия потолка валентной зоны, $\omega_{l}(\Theta)-$ энергия локального уровня, определяемая из уравнения

$$
\omega-\varepsilon_{a}(\Theta)-\Lambda(\omega)=0, \quad|\omega| \leq E_{g} / 2
$$

Легко показать, что учет дипольного отталкивания ведет к уменьшению величины заряда адатомов. Действительно, при $Z_{a}(\Theta)>0$ уровень адатома $\varepsilon_{a}(\Theta)$ сдвигается вниз по шкале энергий, что ведет к возрастанию значений $n_{v}(\Theta), n_{l}(\Theta)$ и, следовательно, к убыли $Z_{a}(\Theta)$. Если же $Z_{a}(\Theta)<0$, уровень $\varepsilon_{a}(\Theta)$ сдвигается по энергии верх, вследствие чего значения $n_{v}(\Theta), n_{l}(\Theta)$ и $\left|Z_{a}(\Theta)\right|$ убывают.

\section{3. Адсорбция на трехмерном полупроводнике}

Для плотности состояний трехмерной (3D) полупроводниковой подложки воспользуемся моделью Халдейна-Андерсона $[2,3]: \quad \rho_{\mathrm{SC}}(\omega)=\rho=\mathrm{const} \quad$ для $|\omega| \geq E_{g} / 2$ (зоны разрешенных состояний) и $\rho_{\mathrm{SC}}(\omega)=0$ при $|\omega|<E_{g} / 2$ (запрещенная зона), где за нуль энергии принят центр запрещенной зоны $E_{g}$. При этом функция сдвига

$$
\Lambda_{\mathrm{SC}}(\omega)=(\Gamma / \pi) \ln \left|\left(\omega-E_{g} / 2\right) /\left(\omega+E_{g} / 2\right)\right|,
$$

где $\Gamma=\pi V^{2} \rho=$ const. Обобщая результаты работы [10] на конечные покрытия, можем записать:

$$
\begin{gathered}
n_{v}(\Theta)=\frac{1}{\pi} \operatorname{arcctg} \frac{\varepsilon_{a}(\Theta)+R}{\Gamma}, \quad R=\frac{E_{g}}{2} \sqrt{1+\frac{4 \Gamma}{\pi E_{g}}} \\
n_{l}(\Theta)=v_{l}(\Theta) \Theta_{H}\left(E_{\mathrm{F}}-\omega_{l}(\Theta)\right) \\
v_{l}(\Theta)=\left(1+\frac{\Gamma}{\pi} \frac{E_{g}}{\left(E_{g} / 2\right)^{2}-\omega_{l}^{2}(\Theta)}\right)^{-1}
\end{gathered}
$$

где $\Theta_{H}\left(E_{\mathrm{F}}-\omega_{l}(\Theta)\right)-$ функция Хэвисайда. Связь вкладов зонных и локальных состояний с парамет-
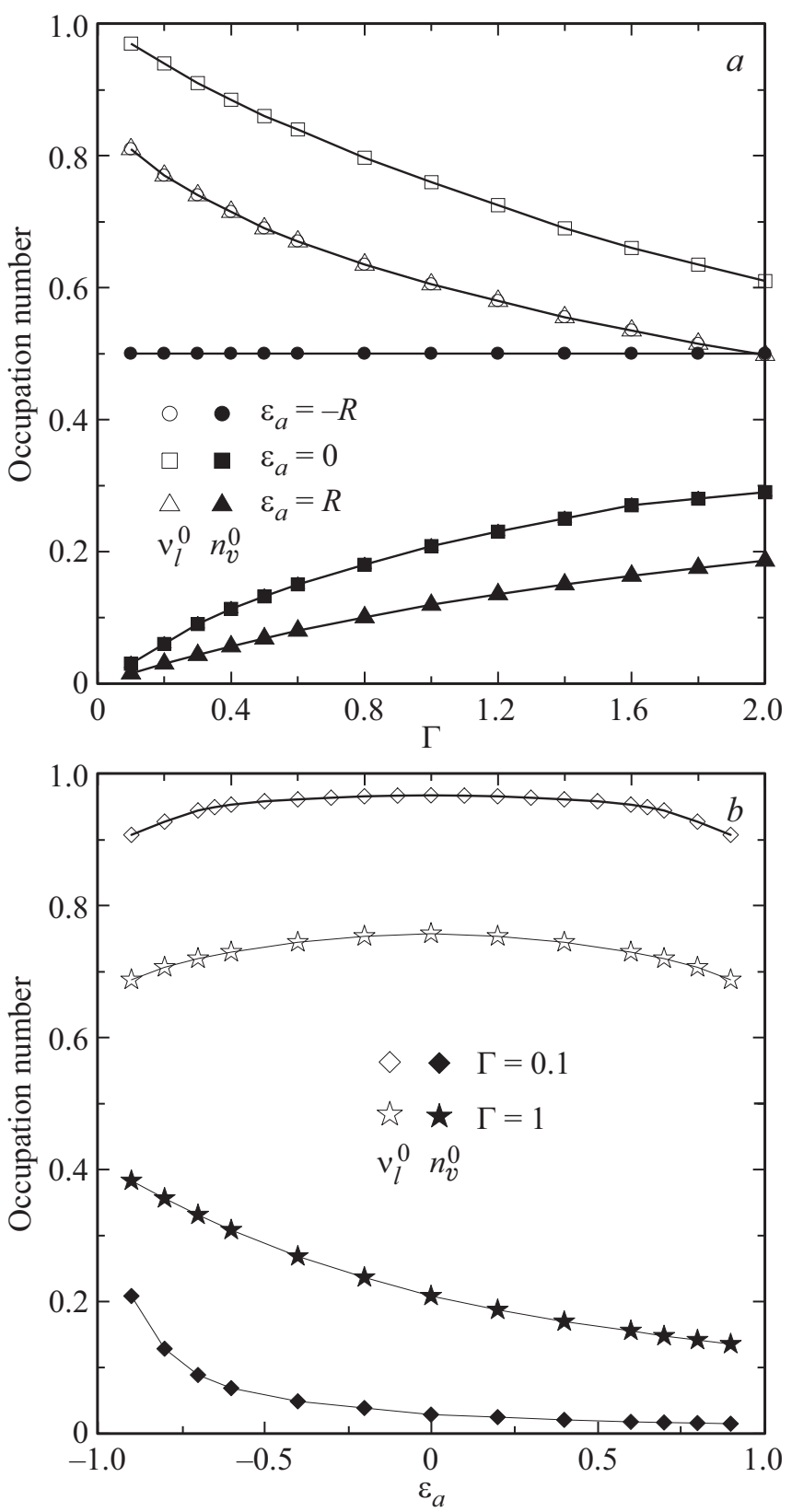

Зависимости вкладов состояний валентной зоны $n_{v}^{0}$ (темные символы) и локальных состояний $v_{l}^{0}$ (светлые символы) в число заполнения одиночного адатома $n_{a}^{0}$ от $(a)$ полуширины $\Gamma$ и $(b)$ энергии $\varepsilon_{a}$ квазиуровня. Все энергетические величины представлены в ед. $E_{g} / 2$.

рами задачи для нулевых покрытий демонстрируется на рисунке. Из рисунка следует, во-первых, что зависимости $n_{v}^{0} \equiv n_{v}(0) \quad$ и $\quad v_{l}^{0} \equiv v_{l}(0)$ являются монотонными функциями параметров $\Gamma$ и $\varepsilon_{a}$. Во-вторых, для представленных на рисунке значений $\Gamma$ и $\varepsilon_{a}$ сумма $n_{v}^{0}+v_{l}^{0}$ близка к 1 (достаточно резкий рост $n_{v}^{0}+v_{l}^{0}$ имеет место только при $\left.2 \Gamma / E_{g} \rightarrow 0\right)$. В пределе $\left|\varepsilon_{a}+R\right| / \Gamma \gg 1$ получаем из (5) и (6) соответственно $n_{v}^{0} \approx\left[1-1 \operatorname{sgn}\left(\varepsilon_{a}+R\right)\right] / 2+\Gamma / \pi\left(\varepsilon_{a}+R\right)$ и $v_{l}^{0} \approx\left(2 \pi E_{g} / \Gamma\right) \exp \left[-\pi\left|\varepsilon_{a}\right| / \Gamma\right]$. В обратном случае 
$\left|\varepsilon_{a}+R\right| / \Gamma \ll 1$ получаем

$$
n_{v}^{0} \approx 1 / 2-\Gamma / \pi\left(\varepsilon_{a}+R\right)
$$

И

$$
\begin{aligned}
v_{l}^{0} \approx & \left(1+4 \Gamma / \pi E_{g}\right)^{-1} \\
& \times\left(1-\frac{16 \Gamma \varepsilon_{a}^{2}}{E_{g}^{3}\left(1+4 \Gamma / \pi E_{g}\right)\left(1+4 \Gamma / E_{g}\right)^{2}}\right) .
\end{aligned}
$$

Переходя к конечным покрытиям, рассмотрим два частных случая. Как вытекает из (6), при $E_{\mathrm{F}}<\omega_{l}(\Theta)$ имеем $n_{a}(\Theta)=n_{v}(\Theta)$ и $Z_{a}(\Theta)=Z_{v}(\Theta)$, так как локальные состояния пусты во всем диапазоне изменений $\Theta$. Тогда задача сводится к решению уравнения (5), в котором $\varepsilon_{d i p}(\Theta)=\Theta^{3 / 2} \xi Z_{v}(\Theta)$, где $Z_{v}(\Theta)=1-n_{v}(\Theta)$ для адатомов-доноров и $Z_{v}(\Theta)=-n_{v}(\Theta)$ для адатомовакцепторов. (Отметим, что уравнение (5) совпадает с уравнением (1) при замене $\varepsilon_{a}-E_{\mathrm{F}}$ на $\left.\varepsilon_{a}+R\right)$. К аналогичному результату приходим в пределе $\left|\varepsilon_{a}(\Theta)\right| \gg \max \left\{E_{g} / 2, \Gamma\right\}$. Действительно, положив $\omega_{l}(\Theta)= \pm E_{g} / 2 \mp \delta(\Theta)$, где $\delta(\Theta) \ll E_{g} / 2$, получим

$$
v_{l}(\Theta) \approx\left(2 \pi E_{g} / \Gamma\right) \exp \left[-\pi\left|\varepsilon_{a}(\Theta)\right| / \Gamma\right] \ll 1
$$

Так как в том же пределе $n_{v}(\Theta) \sim 1$, имеем $n_{v}(\Theta) \gg v_{l}(\Theta)$. Для адатома-донора с точностью до второго порядка по $\gamma=\Gamma /\left|\varepsilon_{a}+R\right| \ll 1$ при $\varepsilon_{a}(\Theta)<0$ получим $Z_{v}(\Theta) \approx \alpha(\Theta)$, где $\alpha(\omega)=(\gamma / \pi)$ $\times\left(1-\Theta^{3 / 2} \gamma \xi /\left|\varepsilon_{a}+R\right|\right)$. При тех же условиях для адатома-акцептора имеем $Z_{v}(\Theta) \approx-\alpha(\Theta)$.

Обратимся теперь к противоположному предельному случаю, когда $n_{v}(\Theta) \ll v_{l}(\Theta)$, так что при $\omega_{l}(\Theta)<E_{\mathrm{F}}$ имеем $n_{a}(\Theta) \approx n_{v}(\Theta)$ и $Z_{a}(\Theta) \approx Z_{l}(\Theta)$. В этом случае задача сводится к решению уравнения (6). $\mathrm{B}$ пределе $\left|\varepsilon_{a}(\Theta)\right| \ll \min \left\{E_{g} / 2, \Gamma\right\}$ получаем $\omega_{l}(\Theta) \approx$ $\approx \varepsilon_{a}(\Theta) /\left(1+2 \Gamma / E_{g}\right) \quad$ и $\quad n_{l}(\Theta) \approx n_{*}-C \Gamma \varepsilon_{a}^{2}(\Theta) / E_{g}^{3}$, где $\quad n_{*}=\left(1+4 \Gamma / \pi E_{g}\right)^{-1} \quad$ и $\quad C=16 / n_{*}^{2}\left(1+4 \Gamma / E_{g}\right)^{2}$. Положив для простоты $\varepsilon_{a}=0$, для адатома-донора находим $Z_{l}(\Theta) \approx Z_{*}(1-\beta(\Theta))$, где $Z_{*}=1-n_{*} \quad$ и $\beta(\Theta)=C Z_{*} \Gamma \xi^{2} \Theta^{3 / 2} / E_{g}^{3}$, а для адатома-акцептора $Z_{*}=-n_{*}\left(1-\beta(\Theta) n_{*}\right)$.

Итак, в рассмотренных предельных случаях с ростом $\Theta$ величина заряда уменьшается, что совпадает с выводами теории адсорбции на металлах [1-3]. В общем случае необходимо решать систему уравнений (5) и (6) методом итераций для каждого значения $\Theta$.

\section{4. Адсорбция на двумерном полупроводнике}

Рассмотрим теперь адсорбцию на графеноподобных соединениях (ГПС) [9,11], являющихся двумерными (2D) аналогами классических трехмерных полупроводников, рассмотренных в предыдущем разделе. Для описания плотности состояний ГПС-подложки воспользуемся низкоэнергетическим (линейном по $\omega$ ) приближением, в рамках которого $\rho_{\mathrm{GLC}}(\omega)=2|\omega| / \xi^{2}$ при $\sqrt{\xi^{2}+\Delta^{2}} \geq|\omega| \geq \Delta$ и $\rho_{\mathrm{GLC}}(\omega)=0$ при $|\omega|<\Delta$ и $|\omega|>\sqrt{\xi^{2}+\Delta^{2}}$, где $2 \Delta-$ ширина запрещенной зоны (щели), $\xi=\sqrt{2 \pi \sqrt{3}} t-$ нормировочный множитель, $t$ - энергия перехода между $p_{z}$-орбиталями ближайших соседями, начало отсчета энергии помещено в центр щели $[9,11]$. (При $\Delta=0$ получаем плотность состояний свободного графена). Такой плотности состояний соответствует функция сдвига

$$
\Lambda_{\mathrm{GLC}}(\omega)=2 \omega(V / \xi)^{2} \ln \left|\left(\omega^{2}-\Delta^{2}\right) /\left(\omega^{2}-\Delta^{2}-\xi^{2}\right)\right| .
$$

Обобщая оценки работы [9] на случай конечных покрытий, представим вклад состояний валентной зоны в суммарное число заполнения в виде

$$
\begin{aligned}
& n_{v}(\Theta) \approx \frac{1}{\pi} \\
& \times\left(\operatorname{arctg} \frac{\omega_{r}(\Theta)+\sqrt{\xi^{2}+\Delta^{2}}}{2 \pi v\left|\omega_{r}(\Theta)\right|}-\operatorname{arctg} \frac{\omega_{r}(\Theta)+\Delta}{2 \pi v\left|\omega_{r}(\Theta)\right|}\right),
\end{aligned}
$$

где $v=2 V^{2} / \xi^{2}, \omega_{r}(\Theta)$ - энергия резонансного уровня, равная корню уравнения $\omega-\varepsilon_{a}(\Theta)-\Lambda_{\mathrm{GLC}}(\omega)=0$ в интервале $\left(-\sqrt{\xi^{2}+\Delta^{2}}-\Delta\right)$ и соответствующая валентной зоне (см. подробности в [9]). В предположении, что $\xi \gg \max \left\{\Delta, V, \omega_{r}(\Theta)\right\}$, получаем уравнение $(5)$, где $\Gamma$ заменяется на $2 \pi v\left|\omega_{r}(\Theta)\right|$ и $R$ на $\Delta$. Легко показать, что при $\xi \gg \max \left\{\Delta, V, \omega_{r}(\Theta)\right\}$ с точностью до $v$ имеем $n_{v}(\Theta) \approx 1-2 v$, а значения $n_{l}(\Theta)$ (как и в предыдущем полупроводниковом случае) экспоненциально малы, т. е. $n_{v}(\Theta) \gg v_{l}(\Theta)$. Так как одним из условий полученного решения является выполнения неравенства $\xi \gg \Delta$, этот случай можно отнести к узкощелевым ГПС.

Рассмотрим теперь другой предельный случай, когда $n_{v}(\Theta) \ll v_{l}(\Theta)$ и $\omega_{l}(\Theta)<E_{\mathrm{F}}$, что может быть реализовано при $\left|\varepsilon_{a}(\Theta)\right| \ll \Delta$, или, принимая, как и в предыдущем случае для простоты $\varepsilon_{a}=0$, получим $\left|\varepsilon_{d i p}(\Theta)\right| \ll \Delta$. Последнее неравенство тем легче удовлетворить, чем выше значение отношения $\Delta / \zeta$. Таким образом, рассматриваемый случай можно отнести к широкощелевым ГПС.

\section{5. Обсуждение результатов и заключительные замечания}

В настоящей работе мы предложили простые модельные схемы учета диполь-дипольного отталкивания адатомов при адсорбции на трех- и двумерных полупроводниковых подложках. При этом каких-либо качественных различий между переходом заряда при адсорбции на 3D- и 2D-полупроводниках не наблюдается. Последнее обстоятельство требует пояснений.

В рамках использованного в настоящей работе подхода, легко выявить причину сходства адсорбционных характеристик для 3D- и 2D-полупроводниковых подложек, усложнив модель плотности 3D-состояний Халдейна-Андерсона и упростив использованную здесь 
модель плотности 2D-состояний. В первом случае следует положить $\rho_{\mathrm{SC}}(\omega)=$ const при $W+E_{g} / 2 \geq|\omega| \geq E_{g} / 2$ и $\rho_{\mathrm{SC}}(\omega)=0$ при $|\omega|<E_{g} / 2$ и $W+E_{g} / 2<|\omega|$, где $W$ - ширина разрешенных зон (еще более усложняя модель, для валентной зоны можно ввести $W_{V}$, для зоны проводимости $\left.-W_{C}\right)$. С другой стороны, в случае ГПС плотность состояний можно представить в виде $\rho_{\mathrm{GLC}}(\omega)=\left(\Delta+\sqrt{\xi^{2}+\Delta^{2}}\right) / \xi^{2}=$ const при $\sqrt{\xi^{2}+\Delta^{2}} \geq|\omega| \geq \Delta$ и $\rho_{\mathrm{GLC}}(\omega)=0$ при $|\omega|<\Delta$ и $|\omega|>\sqrt{\xi^{2}+\Delta^{2}}$.

Можно поступить и по-другому, приняв за модель плотности состояний 3D-подложки параболическую модель вида

$\rho_{\mathrm{SC}}(\omega)=A \begin{cases}\sqrt{\omega-E_{g} / 2}, & E_{g} / 2<\omega \leq W+E_{g} / 2, \\ \sqrt{-\omega-E_{g} / 2}, & -W-E_{g} / 2 \leq \omega<-E_{g} / 2, \\ 0, & |\omega| \leq E_{g} / 2,|\omega|>W+E_{g} / 2,\end{cases}$

где $A-$ коэффициент [12]. Такая плотность состояний качественно близка к функции $\rho_{\mathrm{GLC}}(\omega)$, использованной в настоящей работе.

В общем случае плотность состояния 2D-системы (в отличие от 3D) обладает расходимостями, или особенностями Ван Хова (см., например, модель ВегераЛаббе-Фриделя в [13] или плотность состояний графена [14]). При вычислении перехода заряда между подложкой и адчастицей (как и при нахождении энергии адсорбции) вследствие интегрирования качественные различия между 3D- и 2D-адсорбционными системами, связанные с расходимостями, исчезают.

Мы показали также, что, по сравнению со случаем адсорбции на металле, специфика задачи об адсорбции на 3D- и 2D-полупроводнике сводится к учету локальных состояний. Отметим, что эффект влияния температуры на переход заряда в системах со щелью, который рассматривался в работе [15], сводится, в первую очередь, к вопросу о заполнении локальных состояний. Следует подчеркнуть, что по сравнению с адсорбцией на металлах $[1,2,4,7,16]$, адсорбция даже на классических трехмерных полупроводниках изучена крайне слабо как с точки зрения эксперимента, так и теории. Особенно ощущается отсутствие данных по зависимости электронного состояния адслоя от типа проводимости полупроводника (см. обсуждение данного вопроса в [17]). Именно поэтому в настоящей работе мы ограничились простыми качественными оценками.

\section{Конфликт интересов}

Авторы заявляют об отсутствии конфликта интересов.
[2] С.Ю. Давыдов. Теория адсорбции: метод модельных гамильтонианов. Изд-во СПбГЭТУ „ЛЭТИ“, СПб (2013). 235 c. twirpx.com/file/1596114/.

[3] С.Ю. Давыдов. А.А. Лебедев, О.В. Посредник. Элементарное введение в теорию нано-систем. Изд-во „Лань“, СПб (2014).

[4] J.P. Muscat, D.M. Newns. J. Phys. C 7, 2630 (1974).

[5] Ч. Киттель. Квантовая теория твердых тел. Наука, М. (1967). Гл. 18.

[6] Т. Мория Спиновые флуктуации в магнетиках с коллективизированными электронами. Мир, М. (1988). Гл. 6.

[7] S.G Davison., K.W. Sulston. Green-Function Theory of Chemisorption. Berlin-Heidelberg, Springer (2006).

[8] C.E. Carrol, J.W. May. Surf. Sci. 29, 60 (1972).

[9] С.Ю. Давыдов. ФТП 51, 226 (2017).

[10] С.Ю. Давыдов, С.В. Трошин. ФТТ 49, 8, 1508 (2007).

[11] С.Ю. Давыдов. ФТТ 58, 779 (2016).

[12] С.Ю. Давыдов. ЖТФ 84, 155 (2014).

[13] С.В. Вонсовский, Ю.А. Изюмов, Э.З. Курмаев. Сверхпроводимость переходных металлов, их сплавов и соединений. Наука, М. (1977). Гл. V.

[14] A.H. Castro Neto, F. Guinea, N.M.R. Peres, K.S. Novoselov, A.K. Geim. Rev. Mod. Phys. 81, 109 (2008).

[15] С.Ю. Давыдов. ЖТФ 86, 145 (2016).

[16] Л.А. Большов, А.П. Напартович, А.Г. Наумовец, А.Г. Федорус. УФН 122, 125 (1977).

[17] С.Ю. Давыдов, В.О. Посредник. ЖТФ 87, 635 (2017).

Редактор Т.Н. Василевская

\section{Список литературы}

[1] О.М. Браун, В.К. Медведев. УФН 157, 631 (1989). 